Georgian Mathematical Journal

1(1994), No. 5, 479-486

\title{
GENERALIZED SIERPINSKI SETS
}

\author{
A. KHARAZISHVILI
}

\begin{abstract}
The notion of a Sierpinski topological space is introduced and some properties of such spaces connected with Borel measures are considered.
\end{abstract}

We assume that all topological spaces $E$ to be considered below possess the following property: any singleton in $E$ is a Borel subset of $E$. In particular, all Hausdorff topological spaces possess this property.

We say that a topological space $E$ is a Luzin space if each $\sigma$-finite continuous, i.e., difffused, measure defined on the Borel $\sigma$-algebra of $E$ is identically zero.

We say that a topological space $E$ is a Sierpinski space if $E$ contains none of Luzin spaces with the cardinality equal to $\operatorname{card}(E)$.

The classical Luzin set on the real line $R$ gives us a nontrivial example of an uncountable Luzin topological space (see, for example, [1]). Similarly, the classical Sierpinski set on $R$ gives us a nontrivial example of an uncountable Sierpinski topological space (see [1]). Therefore Luzin topological spaces (accordingly, Sierpinski topological spaces) may be considered as generalized Luzin sets (accordingly, as generalized Sierpinski sets). Some properties of Luzin and Sierpinski topological spaces are investigated in [2] and [3]. In this paper we investigate some other properties of Sierpinski topological spaces.

It is obvious that the cardinality of any Luzin topological space is strictly majorized by the first measurable (in the broad sense) cardinal number. The following simple proposition gives a characterization of measurable (in the broad sense) cardinal numbers in the terms of Sierpinski topological spaces.

Proposition 1. Let $E$ be the main base set. Then the next two relations are equivalent:

1) $\operatorname{card}(E)$ is a measurable (in the broad sense) cardinal number;

$2)$ the topological space $(E, T)$ is a Sierpinski space for every topology $T$ on the set $E$.

1991 Mathematics Subject Classification. 54H05. 
Proof. Indeed, if $\operatorname{card}(E)$ is a measurable (in the broad sense) cardinal number, then for each set $X \subset E$ with $\operatorname{card}(X)=\operatorname{card}(E)$ there exists a probability continuous measure defined on the family of all subsets of $X$. Therefore for any topology $T$ on the set $E$ the space $(E, T)$ contains none of Luzin spaces with the cardinality equal to $\operatorname{card}(E)$. Thus we see that in this situation the topological space $(E, T)$ is a Sierpinski space. Conversely, let us assume that the topological space $(E, T)$ is a Sierpinski space for every topology $T$ on the set $E$. If we set $T=$ a discrete topology on $E$, then we immediately find that $\operatorname{card}(E)$ is a measurable (in the broad sense) cardinal number.

It is not difficult to verify that if $E$ is a Sierpinski topological space and $X$ is a subspace of $E$ with $\operatorname{card}(X)=\operatorname{card}(E)$, then $X$ too is a Sierpinski topological space.

Proposition 2. Let $E$ be a topological space and let

$$
E=\bigcup_{i \in I} E_{i}
$$

where $\left(E_{i}\right)_{i \in I}$ is a finite family of Sierpinski subspaces of E. Then the topological space $E$ is a Sierpinski space. In particular, the topological sum of any finite family of Sierpinski spaces is also a Sierpinski space.

Proof. Let $X$ be an arbitrary subspace of the space $E$ with $\operatorname{card}(X)=$ $\operatorname{card}(E)$. Assume that $X$ is a Luzin subspace of the space $E$. Since the set of indices $I$ is finite, there exists an index $i \in I$ such that the equality $\operatorname{card}\left(E_{i} \cap X\right)=\operatorname{card}\left(E_{i}\right)=\operatorname{card}(E)$ is fulfilled.

Let us consider the set $E_{i} \cap X$. This set, being a subset of the Luzin topological space $X$, is also the Luzin space. At the same time, this set is a subspace of the topological space $E_{i}$. Therefore we see that the Siepinski topological space $E_{i}$ contains the Luzin topological space $E_{i} \cap X$ with the same cardinality, which is impossible. The obtained contradiction proves the proposition.

We shall ascertain below that the result of Proposition 2, generally speaking, does not hold for topological sums of infinite families of Sierpinski spaces.

Let $E$ be the main base set whose cardinality is not cofinal with the least infinite cardinal number $\omega=\omega_{0}$. We set $T(E)=\{X \subset E: \operatorname{card}(E \backslash X)<$ $\operatorname{card}(E)\} \cup\{\varnothing\}$. It is not difficult to verify that $T(E)$ is a topology in $E$ and the topological space $(E, T(E))$ is a Sierpinski space. It is further easy to ascertain that if $\operatorname{card}(E)=\omega_{\xi+1}$, then any subset of $(E, T(E))$, having the cardinality $\omega_{\xi}$, is discrete. Hence it follows that if $\operatorname{card}(E)=\omega_{\xi+1}$ and the cardinal number $\omega_{\xi}$ is not measurable (in the broad sense), then any subset 
of the space $(E, T(E))$, having the cardinality $\omega_{\xi}$, is a Luzin topological space.

Let us now consider a countable disjoint familiy of sets $\left(E_{n}\right)_{n \in \omega}$ where $(\forall n)\left(n \in \omega \Rightarrow \operatorname{card}\left(E_{n}\right)=\omega_{n+1}\right)$.

Provide each set $E_{n}$ with the topology $T\left(E_{n}\right)$ defined above and denote by $E$ the space which is the topological sum of the family of spaces $\left(E_{n}, T\left(E_{n}\right)\right)_{n \in \omega}$. We assert that $E$ is not a Sierpinski space. Indeed, each space $\left(E_{n}, T\left(E_{n}\right)\right)$ contains a discrete subspace $L_{n}$ with the cardinality equal to $\omega_{n}$. By the well-known result of Ulam the cardinal number $\omega_{n}$ is not measurable (in the broad sense). Therefore $L_{n}$ is a Luzin space. Thus the space $E$ contains the topological sum $L$ of the countable family $\left(L_{n}\right)_{n \in \omega}$ of Luzin spaces. Obviously, $L$ itself is a Lusin space (we have the general fact by virtue of which the topological sum of the family of Luzin spaces is a Luzin space if the cardinality of the set of indices of this family is not measurable in the broad sense). Finally, it is absolutely clear that $\operatorname{card}(L)=\operatorname{card}(E)=\omega_{\omega}$ and thus the topological space $E$ is not a Sierpinski space.

Similar arguments are used to prove

Proposition 3. Let us assume that all cardinal numbers are not measurable in the broad sense (this assumption does not contradict the standard axioms of the modern set theory). Then for any infinite cardinal number a there exists a family $\left(E_{i}\right)_{i \in I}$ of topological spaces such that

1) $\operatorname{card}(I)=a$;

2) each $E_{i}$ is a Sierpinski topological space;

3) the topological sum of the family $\left(E_{i}\right)_{i \in I}$ is not a Sierpinski space.

On the other hand, we would like to note here that by Proposition 1 if $\left(E_{i}\right)_{i \in I}$ is a family of Sierpinski spaces and $\operatorname{card}(I)$ is a measurable (in the broad sense) cardinal number, then the topological sum $\left(E_{i}\right)_{i \in I}$ too is a Sierpinski space.

Example 1. Let $E$ be the main base set and $\Phi$ be a filter in $E$. Consider the topology $T_{\Phi}$ on $E$ associated with the filter $\Phi$. This topology is defined by the equality $T_{\Phi}=\{X: X \in \Phi\} \cup\{\varnothing\}$. It is clear that the topology $T(E)$ considered above is the particular case of the topology associated with the filter. Assume that for the filter $\Phi$ the following conditions hold:

1) for each element $x \in E$ the set $E \backslash\{x\}$ belongs to the filter $\Phi$;

2 ) the filter $\Phi$ is countably complete ( $\omega_{1}$-complete), i.e., $\Phi$ is closed with respect to intersections of arbitrary countable families of its elements.

Now we can see that the topological space $\left(E, T_{\Phi}\right)$ is not a Luzin space, since in this situation there exists a two-valued probability continuous Borel measure on $E$. Assume also that the cardinality of $E$ is not measurable in the broad sense. Then the said topological space $\left(E, T_{\Phi}\right)$ is a Sierpinski 
space if and only if the relation $X \cap Y \neq \varnothing$ is fulfilled for each set $X \subset E$ with $\operatorname{card}(X)=\operatorname{card}(E)$ and for each set $Y \in \Phi$.

Let now $E$ be a topological space. Assume that $E$ is not a Luzin space. Then there naturally arises the question: does the space $E$ contain at least one Sierpinski subspace? It will be shown below that the answer to this question is negative even in the case when the cardinality of the space $E$ is equal to the first uncountable cardinal number $\omega_{1}$. For this we need one auxiliary assertion.

Lemma. Let $\omega_{\alpha}$ be an arbitrary uncountable regular initial order number provided with the standard order topology and $X$ be an arbitrary unbounded subset of $\omega_{\alpha}$. Then there exists an unbounded nonstationary (in $\left.\omega_{\alpha}\right)$ set $Y \subset X$.

Proof. The required set $Y \subset X$ can be readily constructed by the method of transfinite recursion. Indeed, using the regularity of the ordinal number $\omega_{\alpha}$, it is possible to define by transfinite recursion the family of sets $\left(Z_{\xi}\right)_{\xi<\omega_{\alpha}}$ satisfying the following conditions:

1) for any index $\xi<\omega_{\alpha}$ the set $Z_{\xi}$ is an open bounded interval in the ordered set $\omega_{\alpha}$

2) the intersection $Z_{\xi} \cap X$ is not empty for any index $\xi<\omega_{\alpha}$;

3 ) for any indices $\xi<\omega_{\alpha}$ and $\zeta<\omega_{\alpha}$ such that $\xi<\zeta$, the right end-point of the interval $Z_{\xi}$ is strictly less that the left end-point of the interval $Z_{\zeta}$.

Having constructed the family $\left(Z_{\xi}\right)_{\xi<\omega_{\alpha}}$ we choose for each ordinal number $\xi<\omega_{\alpha}$, an element $y_{\xi}$ from the nonempty intersection $Z_{\xi} \cap X$ and set

$$
Y=\bigcup_{\xi<\omega_{\alpha}}\left\{y_{\xi}\right\} .
$$

Now it is not difficult to verify that the set $Y$ is the required one, since it is unbounded and nonstationary in $\omega_{\alpha}$ and is certainly entirely contained in the original set $X$.

Example 2. Let us consider the first uncountable ordinal number $\omega_{1}$ provided with its order topology. Let $\Phi$ be the filter in the topological space $\omega_{1}$ generated by the family of all unbounded closed subsets of this space. Further, let $T_{\Phi}$ be the topology in the set $\omega_{1}$ associated with the filter $\Phi$. Then the topological space $\left(\omega_{1}, T_{\Phi}\right)$ is not a Luzin space. Indeed, if $\mu$ is the standard two-valued probability continuous Borel measure in the space $\omega_{1}$, then the domain of definition of the measure $\bar{\mu}$ (of the usual completion of $\mu$ ) coincides with the Borel $\sigma$-algebra of the topological space $\left(\omega_{1}, T_{\Phi}\right)$. Thus we see that there exist nontrivial $\sigma$-finite Borel measures on the space $\left(\omega_{1}, T_{\Phi}\right)$. Let us ascertain that at the same time the space $\left(\omega_{1}, T_{\Phi}\right)$ does not contain any Sierpinski subspace. Let $X$ be an arbitrary subspace of the space $\left(\omega_{1}, T_{\Phi}\right)$. Without loss of generality it can be assumed that the 
cardinality of $X$ is equal to $\omega_{1}$. Then, according to the above lemma, there exists a nonstationary (in $\omega_{1}$ ) set $Y \subset X$ with the cardinality also equal to $\omega_{1}$. Note now that the subspace $Y$ of the space $X$ is discrete. Taking into consideration the fact that by the result of Ulam the cardinal number $\omega_{1}$ is not measurable in the broad sense, we see that $Y$ is a Luzin subspace of the space $X$. Finally, we ascertain that the topological space $X$ is not a Sierpinski space. Therefore our topological space $\left(\omega_{1}, T_{\Phi}\right)$ does not contain Seirpinski subspaces.

A similar example can evidently be constructed for an arbitrary uncountable regular nonmeasurable (in the broad sense) cardinal number $\omega_{\alpha}$.

For our further consideration we need two simple notions from the general set theory.

Let $E$ be a base set and $D$ be a family of subsets of $E$ covering $E$. We put $\operatorname{cov}(D)=\inf \left\{\operatorname{card}\left(D^{\prime}\right): D^{\prime} \subset D\right.$ and $\left.D^{\prime} \operatorname{covers} E\right\}$.

It is easy to see that the cardinal number $\operatorname{cov}(D)$ is an invariant with respect to bijective mappings. Therefore $\operatorname{cov}(D)$ can be regarded as some cardinal-valued characteristic of the given family $D$.

Let $E$ be a base set again and $D$ be an arbitrary family of subsets of $E$. We put

$$
\operatorname{cof}(D)=\inf \left\{\operatorname{card}\left(D^{\prime}\right): D^{\prime} \subset D \text { and for any set } X \in D\right. \text { there exists }
$$

a set $Z \in D^{\prime}$ such that $X \subset Z$ \}.

The cardinal number $\operatorname{cof}(D)$ is also an invariant with respect to bijective mappings and therefore can be regarded as another cardinal-valued characteristic of the given family $D$. If $D$ is an ideal of subsets of $E$ and $D^{\prime}$ is a subfamily of $D$ such that $(\forall X)\left(X \in D \Rightarrow(\exists Z)\left(Z \in D^{\prime} \& X \subset Z\right)\right)$, then we say that $D^{\prime}$ is a base of the ideal $D$. Clearly, this notion is dual to the well-known notion of a filter base.

We have the following proposition which gives us some sufficient conditions for the existence, in the topological space $E$, of a Sierpinski subspace with the cardinality equal to $\operatorname{card}(E)$.

Proposition 4. Let $E$ be a topological space, $\mu$ be a nonzero $\sigma$-finite continuous Borel measure in $E$ and $D(\mu)$ be the countably additive ideal of subsets of $E$ generated by all $\mu$-measure-zero sets. It is also assumed that the next two conditions are fulfilled for the ideal $D(\mu)$ :

1) if $\operatorname{card}(I)<\operatorname{card}(E)$, then the union of any family $\left(X_{i}\right)_{i \in I} \subset D(\mu)$ does not coincide with the space $E$ (in other words, the equality $\operatorname{cov}(D(\mu))=$ $\operatorname{card}(E)$ is true);

2) there exists a base of the ideal $D(\mu)$ with the cardinality less than or equal to the cardinality of $E$ (in other words, the inequality $\operatorname{cof}(D(\mu)) \leq$ $\operatorname{card}(E)$ is true). 
Then the topological space $E$ contains some Sierpinski subspace $S$ with $\operatorname{card}(S)=\operatorname{card}(E)$.

Proof. Let $\omega_{\alpha}$ be the initial ordinal number corresponding to the cardinality of the given topological space $E$. Fix any base of the ideal $D(\mu)$ with the cardinality less than or equal to $\omega_{\alpha}$. It is obvious that we can represent this base as a familiy of sets $\left(Z_{\xi}\right)_{\xi<\omega_{\alpha}}$. Now, using the method of transfinite recursion, let us define the $\omega_{\alpha}$-sequence $\left(s_{\xi}\right)_{\xi<\omega_{\alpha}}$ of elements of the given space $E$. Assume that for an ordinal number $\xi<\omega_{\alpha}$ we have already defined the partial $\xi$-sequence $\left(s_{\zeta}\right)_{\zeta<\xi}$ of elements of $E$. Consider the set

$$
\left(\bigcup_{\zeta<\xi} Z_{\zeta}\right) \bigcup\left(\bigcup_{\zeta<\xi}\left\{s_{\zeta}\right\}\right) .
$$

Since the condition 1) holds, this set does not coincide with the space $E$. Therefore there exists an element $s_{\xi} \in E$ which does not belong to the mentioned set. In this manner we shall construct the $\omega_{\alpha}$-sequence $\left(s_{\xi}\right)_{\xi<\omega_{\alpha}}$ and, having done so, set

$$
S=\bigcup_{\xi<\omega_{\alpha}}\left\{s_{\xi}\right\} .
$$

It remains for us to verify that the set $S$ is a Sierpinski subspace of the space $E$. Indeed, let $X$ be an arbitrary subset of $S$ such that $\operatorname{card}(X)=$ $\operatorname{card}(S)=\operatorname{card}(E)=\omega_{\alpha}$. Then the procedure of the construction of the set $S$ immediately implies that the set $X$ is not contained in any of the sets $Z_{\xi}\left(\xi<\omega_{\alpha}\right)$. Thus we have the inequality $\mu^{*}(X)>0$ where $\mu^{*}$ denotes an outer measure associated with the given measure $\mu$. The latter fact leads to a conclusion that the topological space $X$ is not a Luzin subspace. Therefore the constructed topological space $S$ is the Sierpinski space.

One may easily note here that if $\operatorname{card}(E)=\omega_{1}$, then the condition 1) in the formulation of Proposition 4 becomes superfluous. The next simple example, on the other hand, shows that if $\operatorname{card}(E)=\omega_{2}$, then this condition plays an essential role.

Example 3. Assume that the relation $2^{\omega_{1}}=\omega_{2}$ is fulfilled. Take two disjoint sets $E_{1}$ and $E_{2}$ such that $\operatorname{card}\left(E_{1}\right)=\omega_{1}, \operatorname{card}\left(E_{2}\right)=\omega_{2}$. Identify the set $E_{1}$ with the ordinal number $\omega_{1}$ and equip $E_{1}$ with the topology $T_{\Phi}$ (see Example 2). Further, equip the set $E_{2}$ with a discrete topology. Denote by $E$ the topological sum of two spaces $E_{1}$ and $E_{2}$. Now it is not difficult to verify that the topological space $E$ satisfies the following conditions:

a) $\operatorname{card}(E)=\omega_{2}$;

b) there exists a probability continuous Borel measure $\mu$ in the space $E$ such that $\operatorname{cof}(D(\mu)) \leq \omega_{2}$;

c) the space $E$ does not contain any Sierpinski subspace. 
Example 4. Let $\omega_{\alpha}$ be an arbitrary uncountable regular initial ordinal number not measurable in the broad sense. Equip $\omega_{\alpha}$ with its order topology and denote by $\Phi$ the filter in $\omega_{\alpha}$ generated by the family of all closed unbounded subsets of $\omega_{\alpha}$. Further, set $E=\omega_{\alpha}$ and equip $E$ with the topology $T_{\Phi}$. Then the topological space $E$ satisfies the following conditions:

a) $\operatorname{card}(E)=\omega_{\alpha}$;

b) there exists a probability continuous Borel measure $\mu$ in the space $E$ such that $\operatorname{cov}(D(\mu))=\omega_{\alpha}$;

c) the space $E$ does not contain any Sierpinski subspace.

Thus Example 4 shows us that the condition 2) in the formulation of Proposition 4 also plays an essential role and cannot be omitted.

Example 5. Let $E$ be a metric space with the cardinality continuum $2^{\omega}$. It is clear that if the cardinality continuum is measurable in the broad sense, then any subspace $X$ of $E$ with $\operatorname{card}(X)=\operatorname{card}(E)$ is a Sierpinski topological space (in particular, $E$ is not a Luzin topological space). Now let us consider some situation when the cardinality continuum is not measurable in the broad sense. Assume that Martin's axiom holds and that the given metric space $E$ is not a Luzin space. Then one can establish that there always exists a subset $S$ of $E$ satisfying the next two conditions:

a) $\operatorname{card}(S)=\operatorname{card}(E)=2^{\omega}$;

b) $S$ is a Sierpinski subset of the space $E$.

The existence of such a space $S \subset E$ is proved in [3]. Note that the proof is essentially based on the following well-known fact: every $\sigma$-finite Borel measure defined in the space $E$ is concentrated on some separable subspace of $E$ (in other words, for every $\sigma$-finite Borel measure given in the space $E$ there exists a separable support in $E$ ).

Example 6. Let $E$ be again a metric space with the cardinality continuum $2^{\omega}$. Assume that the continuum hypothesis holds and that $E$ is not a first category space. Then there always exists a subset $L$ of $E$ satisfying the next two conditions:

a) $\operatorname{card}(L)=\operatorname{card}(E)=2^{\omega}$;

b) $L$ is a Luzin subspace of the space $E$.

The existence of such a set $L \subset E$ is also proved in [3]. To establish this result we need to consider two cases. We begin by assuming that the space $E$ is not separable. Then there exists a discrete set $L \subset E$ such that $\operatorname{card}(L)=\omega_{1}=2^{\omega}$. As we know, the least uncountable cardinal number $\omega_{1}$ is not measurable in the broad sense. Therefore the subspace $L$ of $E$ is a Luzin topological space with the cardinality continuum. Now assume $E$ to be a separable metric space. In that case every $\sigma$-finite continuous Borel measure defined in the space $E$ is concentrated on a first category subset of $E$. Using the classical transfinite construction due to Luzin (see, 
for instance, [1]), we can define a set $L \subset E$ with the cardinality continuum such that we shall have the inequality $\operatorname{card}(L \cap X) \leq \omega$ for each first category set $X \subset E$.

Now it is not difficult to verify that such a set $L$ is a Luzin subspace of our space $E$.

From the foregoing arguments it also follows that any nonseparable metric space $E$ contains an uncountable Luzin subspace.

Finally, let us formulate one unsolved problem concerning Sierpinski topological spaces.

Problem. Give a characterization of Sierpinski spaces in purely topological terms.

Remark. In the literature the term "Luzin topological space" is used in other senses as well. For example, Luzin topological spaces in the sense of N. Bourbaki coincide with Borel subsets of complete separable metric spaces while in the modern set-theoretical topology Luzin topological spaces are uncountable Hausdorff spaces which contain neither isolated points nor uncountable first category subsets.

\section{REFERENCES}

1. J. Oxtoby, Measure and Category, Springer-Verlag, New York-Heidelberg-Berlin, 1971.

2. A.B. Kharazishvili, Luzin spaces and Bernstein sets. (Russian) Bull. Acad. Sci. Georgian SSR, 138(1990), No.1, 25-28.

3. - Remark on Luzin sets and Sierpinski sets. (Russian) Bull. Acad. Sci. Georgia, 143(1991), No.3, 237-240.

(Received 03.06.1993)

Author's address:

I. Vekua Institute of Applied Mathematics of Tbilisi State University

2, University St., Tbilisi 380043

Republic of Georgia 